\title{
基于多分支并行空洞卷积的多尺度目标检测算法
}

\author{
袁帅，王康，单义，杨金福* \\ (北京工业大学信息学部 北京 100124) \\ (jfyang@bjut.edu.cn)
}

\begin{abstract}
摘 要: 针对现有的目标检测算法在提取特征时往往仅使用单一尺度大小的卷积核, 忽略了不同尺度特征感受野的 差异，从而影响网络对不同尺度目标的检测效果的问题，提出一种基于多分支并行空洞卷积的多尺度目标检测算法. 首先，采用基础网络 VGG-16 对待检测图像进行特征提取; 其次，在网络的低层引人多分支并行空洞卷积，对不同扩 张率的空洞卷积进行融合，从而获取多尺度特征信息，提高网络对不同尺度特征的提取能力; 然后，采用非局部化 结构整合特征的全局空间信息，进而增强上下文信息；最后，在不同尺度大小的特征图上执行目标的检测与定位任 务. 在 PASCAL VOC 数据集和 MS COCO 数据集上的实验结果表明, 所提算法能有效地提高网络对不同尺度目标的 检测准确率, 对小目标检测效果有明显改善.
\end{abstract}

关键词: 深度学习; 目标检测; 空洞卷积; 多尺度特征图

中图法分类号: TP391.41 DOI: 10.3724/SP.J.1089.2021.18529

\section{Multi-Scale Object Detection Method Based on Multi-Branch Parallel Dilated Convolution}

\author{
Yuan Shuai, Wang Kang, Shan Yi, and Yang Jinfu* \\ (Faculty of Information Technology, Beijing University of Technology, Beijing 100124)
}

\begin{abstract}
Existing object detection algorithms only use a fixed size convolution kernel when extracting features, ignoring the difference in the receptive field of different scale features, which affects the detection effect of different scale objects. To solve this problem, a multi-scale object detection network based on multi-branch parallel dilated convolution is proposed. Firstly, the basic network VGG-16 is used to extract the features of the image. Secondly, a multi-branch parallel dilated convolution is designed to extract multi-scale features to improve object detection ability of the network. Then, a non-local block is employed to integrate the global spatial information and enhance the context information. Finally, the object detection and location tasks are performed on feature maps with different scales. Experimental results on PASCAL VOC and MS COCO datasets demonstrate that the proposed method can effectively improve the detection accuracy of different scale objects and clearly improve the detection accuracy of small objects.
\end{abstract}

Key words: deep learning; object detection; dilated convolution; multi-scale feature map

目标检测是图像处理和计算机视觉中的重要 研究方向之一. 随着深度学习的发展, 提出了越来
越多的基于深度学习的目标检测算法, 并取得了 较好的检测结果. 现实场景中往往同时存在不同

收稿日期: 2020-08-01; 修回日期: 2021-04-08. 基金项目: 国家自然科学基金(61973009); 北京市自然科学基金(4182009). 袁帅 (1994一), 男, 硕士研究生, 主要研究方向为机器人环境感知; 王康 (1994一), 男, 硕士研究生, 主要研究方向为机器人视觉环境感知; 单义(1992-), 男, 硕士研究生, 主要研究方向为深度学习、计算机视觉; 杨金福(1977一), 男, 博士, 教授, 博士生导师, CCF 会员, 论文通讯作者, 主要研究方向为智能服务机器人、计算机视觉、模式识别. 
尺度大小的物体, 由于图像中占比较小的目标分 辨率低、携带的信息较少、与其他大小的物体尺度 差异大，导致现有的目标检测算法对小物体的检 测效果不尽人意. 因此，提高网络对不同尺度目标 的检测能力仍然是一项具有挑战性的任务.

目前，基于深度学习的目标检测算法主要分 为 2 类：一类是基于候选区域的目标检测算法，也 称为两阶段算法; 另一类是基于回归的目标检测 算法，也称为单阶段算法. 基于候选区域的目标检 测算法首先产生一系列候选区域，然后对其进行分 类，从而得到检测结果. 其中，典型代表是基于候 选区域的卷积神经网络(region-convolutional neural networks，R-CNN) 系列算法，如 R-CNN 算法 ${ }^{[1]}$, Fast R-CNN算法 ${ }^{[2]}$ 和 Faster R-CNN算法 ${ }^{[3]}$ 等。这类 算法的候选区域仅由最后一层特征经过池化得到, 限制了候选区域的大小，在场景中存在尺度变化 大的物体时检测效果较差. 基于回归的目标检测 算法不需要产生候选区域，经过单次检测即可直 接得到最终的检测结果, 它以 YOLO(you only look once)算法 ${ }^{[4]}$ 和单次多尺度检测器 (single shot multibox detector, SSD)算法 ${ }^{[5]}$ 为代表. YOLO 算法是第 1 个基于回归的目标检测算法，将特征图等分为 $n \times n$ 的网格，每个网格负责检测一个物体，当一个 网格中存在多个目标时，会产生漏检的问题.SSD 算法采用针框替代 YOLO 算法中的网格进行检测, 改进了 YOLO 算法漏检的问题. 为了检测不同尺 寸的目标, SSD 算法分别在细节信息丰富的低层特 征和语义信息丰富的高层特征进行检测。但是由 于 SSD 算法只使用低层特征检测小目标，未能充 分利用上下文信息，导致检测到的物体出现局部 缺失等问题.

为了提高网络对不同尺度目标的检测效果, 许多学者对上述结构进行了改进. 黄继鹏等 ${ }^{[6]}$ 参考 SSD 算法多尺度检测的思想设计了多尺度 Faster R-CNN 检测算法, 采用网络中的不同特征层检测 不同尺度的目标，但是由于候选区域的限制，导致 其在检测小目标时效果较差. 李晓光等 ${ }^{[7]}$ 改进了多 尺度 Faster R-CNN 候选区域的生成方式，可以在 不同尺度的特征图上生成不同大小的候选区域, 提升了 Faster R-CNN 算法对不同尺度目标的检测 效果. 以上基于 Faster R-CNN 的算法虽然进行了 多尺度检测，但是在检测小目标时只使用低层特 征, 忽略了高层特征含有的语义信息，导致小目标 的检测效果差. 同时，由于基于候选区域的目标检 测算法需要产生候选区域, 导致检测速度较慢. 基
于回归的目标检测算法可以满足实时性的要求, 因此, 越来越多的学者选择在 SSD 算法的基础上 增强语义信息，提高网络对不同尺度目标的检测 效果. Lin 等 ${ }^{[8]}$ 提出了一种具有自顶向下结构的特 征金字塔网络，增强低层特征的语义信息. 同样 地，张思宇等 ${ }^{[9]}$ 通过将高层特征进行反卷积与相邻 的低层特征进行逐像素相加, 丰富小目标的特征 表达. Cui 等 ${ }^{[10]}$ 认为使用相邻特征层的信息较为类 似，引人了低层特征和高层特征的跨级连接，但对 不同目标的检测效果同样不尽人意.上述算法在 提取低层特征时只使用了单一尺度大小的卷积核, 忽略了不同尺度特征感受野的差异，没有充分利 用细节信息较多的低层特征，导致网络对小目标 的检测效果差, 影响网络对不同尺度物体的检测 效果. 另外, 以上算法均采用将高层特征与低层特 征融合的方法增强语义信息. 由于高层特征与低 层特征的大小不一致, 因此需要对高层特征图进 行上采样，以保证其与低层特征的大小相同. 但是 在上采样时需要对特征图进行插值, 从而引人额 外的信息, 导致采样后的特征图与原特征图信息 不一致, 影响了网络的检测效果.

基于此，本文提出一种基于多分支并行空洞 卷积的多尺度目标检测算法. 由于 SSD 类算法仅 使用单一尺寸的卷积核提取特征，限制了感受野 的大小，在图像中存在不同尺度大小的物体时检 测效果不佳. 本文对提取低层特征的方法进行了 改进, 在网络的低层引人多分支并行空洞卷积, 利 用不同扩张率的空洞卷积提取不同尺度大小的特 征图, 进而获取不同大小的感受野. 当图像中存在 不同大小的物体时，可以同时提取图像中不同尺 度目标的特征，提高网络的检测能力. 此外, 考虑 图像中的目标通常不是单独存在, 与周围的其他 物体有着不同程度的关联，而采用上采样的方法 增强低层特征上下文语义信息会在特征图中引人 额外的信息, 影响网络的检测效果. 因此, 本文采 用一种非局部化结构计算特征图中某像素点与其 他像素点的相似性, 并进行加权求和后表示该像 素点的特征信息，从而捕获每个像素点特征的全 局信息，达到增强低层特征上下文信息的目的.

\section{1 本文算法}

本文算法模型结构如图 1 所示, 使用基础网络 VGG-16 对待检测图像进行特征提取. 为了便于区 分普通卷积产生的特征层和空洞卷积以及非局部 
化产生的特征层，普通卷积产生的特征层为 $\boldsymbol{F}_{\mathrm{conv}}$, 经过空洞卷积以及非局部化产生的特征层为 $\boldsymbol{F}$. 分别将特征层 $\boldsymbol{F}_{\text {conv4 } 33}$ 和 $\boldsymbol{F}_{\text {conv7 }}$ 经过多分支并行空 洞卷积结构以及非局部化结构, 得到新的特征层 $\boldsymbol{F}_{1}$ 和 $\boldsymbol{F}_{2}$. 考虑特征层 $\boldsymbol{F}_{\mathrm{conv} 7}$ 是由特征层 $\boldsymbol{F}_{\mathrm{conv} 433}$ 进 行卷积得到的, 继续对特征层 $\boldsymbol{F}_{1}$ 进行卷积操作得 到的特征层与 $\boldsymbol{F}_{1}$ 类似，因此选择对特征层 $\boldsymbol{F}_{2}$ 进行 逐层的普通卷积操作，从而得到不同尺度大小的 特征层 $\boldsymbol{F}_{\text {conv8 }}, \boldsymbol{F}_{\text {conv9 }}, \boldsymbol{F}_{\text {conv10 }}$ 和 $\boldsymbol{F}_{\text {conv11 }}$. 最终, 网络
产生 6 层不同尺度大小的特征 $\boldsymbol{F}_{1}, \boldsymbol{F}_{2}, \boldsymbol{F}_{\text {conv8 }}$,

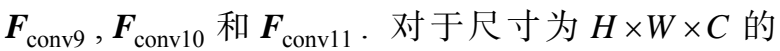
特征图, 使用一系列卷积滤波器产生固定的预测, 包括类别概率值以及包围框与真实包围框之间的 坐标偏移量. 在检测时, 针对不同大小的特征层产 生的大量包围框, 首先通过类别置信度过滤掉属 于背景的包围框和阈值较低的包围框，然后利用 非极大值抑制过滤掉交并比较大的包围框，得到 最终的检测结果.

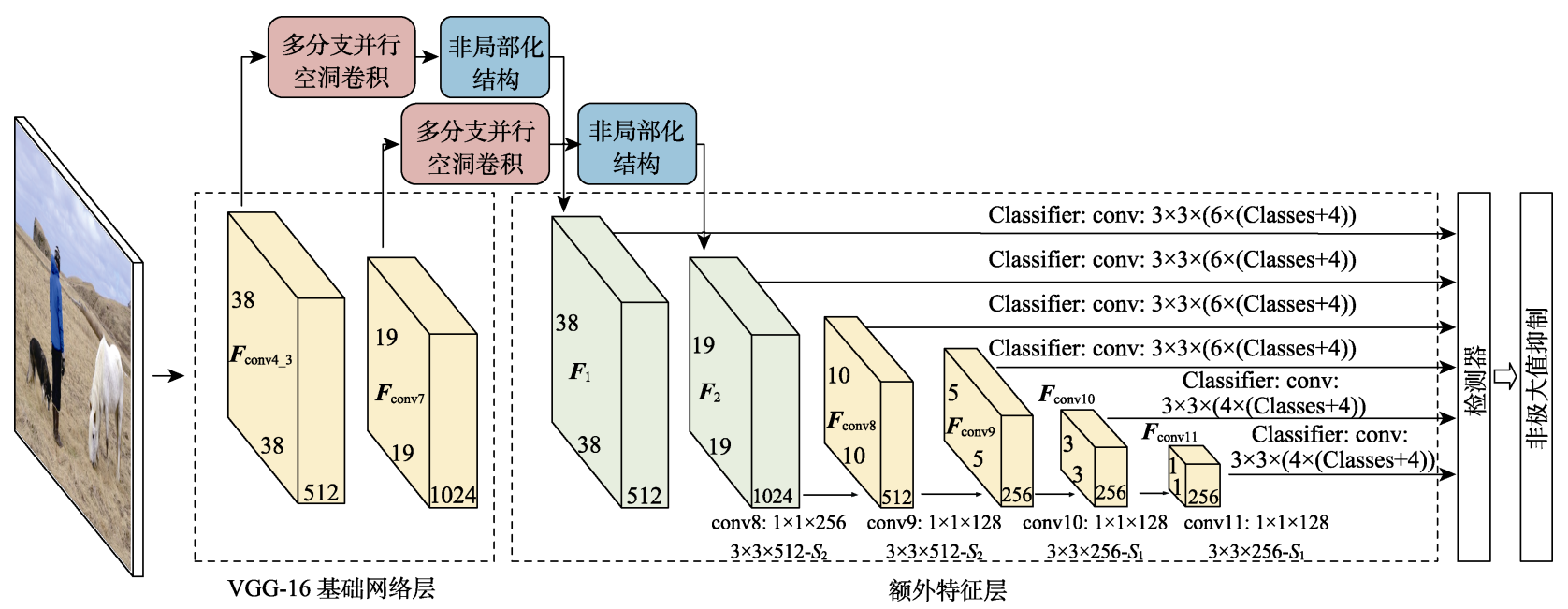

图 1 本文算法模型结构

\section{1 多分支并行空洞卷积}

在卷积神经网络中，卷积核的尺寸决定了卷 积感受野的大小，而不同大小的感受野有利于不 同尺度目标的检测. 如图 $2 \mathrm{~b}$ 所示，只使用 $1 \times 1$ 卷积 核可以产生 $1 \times 1$ 大小的感受野，适合用其检测远处 的红色汽车, 而不适合检测近处的银色汽车. 对于 银色汽车，使用 $5 \times 5$ 大小的感受野更为合适，但对 于远处的红色汽车, $5 \times 5$ 大小的感受野包含目标 之外的树木等信息, 感受野过大, 如图 2c 所示. 本 文旨在根据不同尺寸的目标设计不同大小的感受 野，以获得更加丰富的目标特征，达到提高目标检 测精度的目的, 如图 $2 \mathrm{~d}$ 所示.

在语义分割任务中, 为了更好地对图像中每个 像素点进行分类, 需要较大的感受野, 以提取图像 中较大范围的上下文信息. 其中, 典型代表是 DeepLab 网络 ${ }^{11]}$ 采用的空洞空间金字塔池化(atrous spatial pyramid pooling, ASPP)方法. 如图 3 所示, 通过扩张率分别为 $r=6, r=12, r=18$ 和 $r=24$, 卷积核大小为 $3 \times 3$ 的空洞卷积获取不同大小的感 受野. ASPP 结构在语义分割任务中取得了不错的 效果，但该结构并不适合直接用于目标检测任务.

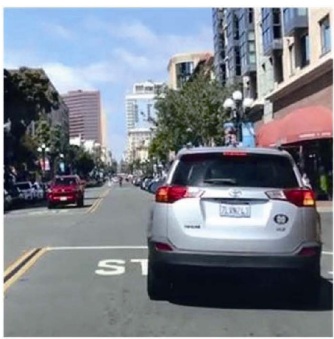

a. 图像

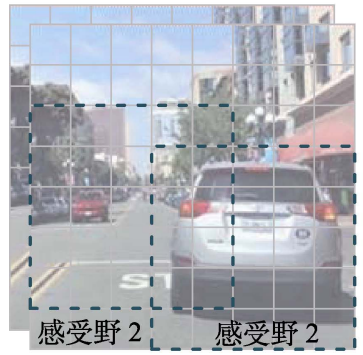

c. $5 \times 5$ 感受野

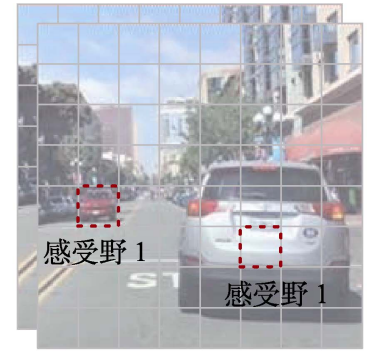

b. $1 \times 1$ 感受野

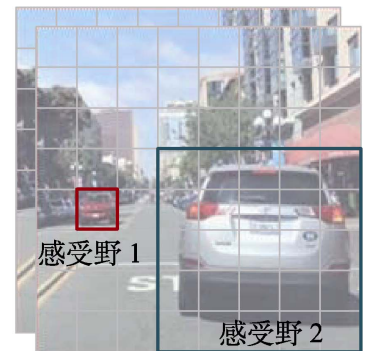

d. 希望的感受野
图 2 不同尺度物体的感受野对比

为了同时提取图像中不同尺度目标的特征，本文 借鉴语义分割任务中常用的 ASPP 结构，提出一种 适用于目标检测任务的多分支并行空洞卷积结构.

对于扩张率为 $r$ 、卷积核大小为 $k$ 的空洞卷积, 


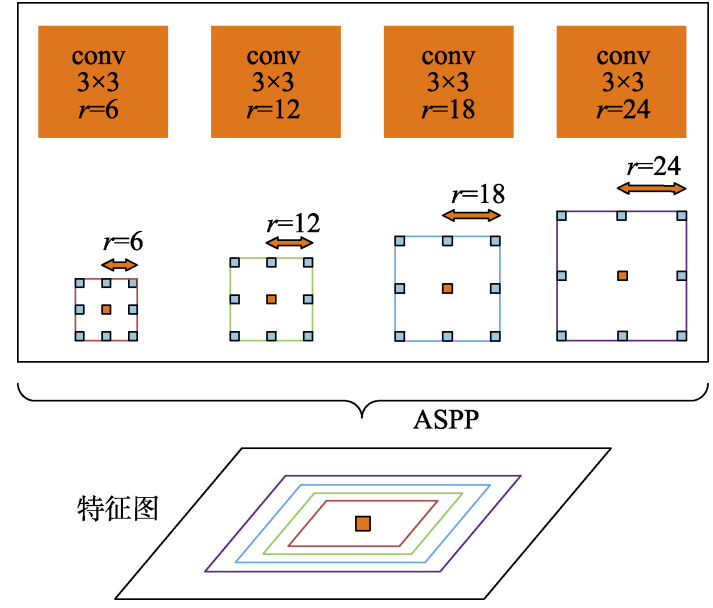

图 3 ASPP 结构 ${ }^{[11]}$

感受野的计算公式为 $((r-1)(k-1)+k+2)^{2}$. 同时, 本文定义扩张率组合为 $R=\left(r_{1}, r_{2}, r_{3}\right)$, 其中, $r_{1}, r_{2}$, $r_{3}$ 分别表示 3 种不同的扩张率. 考虑 ASPP 结构 4 条支路产生的感受野分别为 $15 \times 15 ， 27 \times 27$, $39 \times 39$ 和 $51 \times 51$, 而 $51 \times 51$ 大小的感受野并不适用 于目标检测任务. 因此, 本文将 ASPP 结构的 4 条 分支改进为 3 条分支, 并在实验中将扩张率组合设 置为 $R=(1,3,5)$, 即 3 条分支分别采用卷积核大小 为 $3 \times 3$, 扩展率为 $r=1, r=3$ 和 $r=5$ 的空洞卷积, 产生的感受野大小分别为 $3 \times 3,7 \times 7$ 和 $11 \times 11$.

考虑空洞卷积不会对空洞部分进行采样，导 致提取的信息不具有连续性. 因此, 为了提取空洞 部分的特征信息, 本文提出的多分支并行空洞卷 积结构如图 4 所示. 与 ASPP 结构不同，多分支并 行空洞卷积分别在每条支路的空洞卷积前添加核 大小为 $1 \times 1,3 \times 3$ 和 $5 \times 5$ 的普通卷积, 其中红色像 素点为空洞卷积提取的特征, 其余为普通卷积提 取的特征. 添加普通卷积之后, 空洞部分的信息可 以由普通卷积提取, 在增强信息连续性的同时, 获 取不同大小的感受野，从而提取不同尺度物体的 特征。

多分支并行空洞卷积总体结构如图 5 所示, $\boldsymbol{X}$ 为输人特征, $\boldsymbol{Y}$ 为输出特征. 为了减少计算量, 先采用核大小为 $1 \times 1$ 的普通卷积降低通道数. 采用 不同扩张率的空洞卷积提取不同尺度大小的特征 后, 对其进行通道拼接. 在通道拼接之前, 3 条支 路获取的特征分别为

$$
\begin{gathered}
\boldsymbol{X}_{1}=\boldsymbol{X} * \boldsymbol{C}_{1 \times 1} * \boldsymbol{D}_{r=1} \\
\boldsymbol{X}_{2}=\boldsymbol{X} * \boldsymbol{C}_{1 \times 1} * \boldsymbol{C}_{3 \times 3} * \boldsymbol{D}_{r=3} \\
\boldsymbol{X}_{3}=\boldsymbol{X} * \boldsymbol{C}_{1 \times 1} * \boldsymbol{C}_{5 \times 5} * \boldsymbol{D}_{r=5}
\end{gathered}
$$

其中, $\boldsymbol{X}_{1}, \boldsymbol{X}_{2}$ 和 $\boldsymbol{X}_{3}$ 分别为 3 条支路提取的特征;

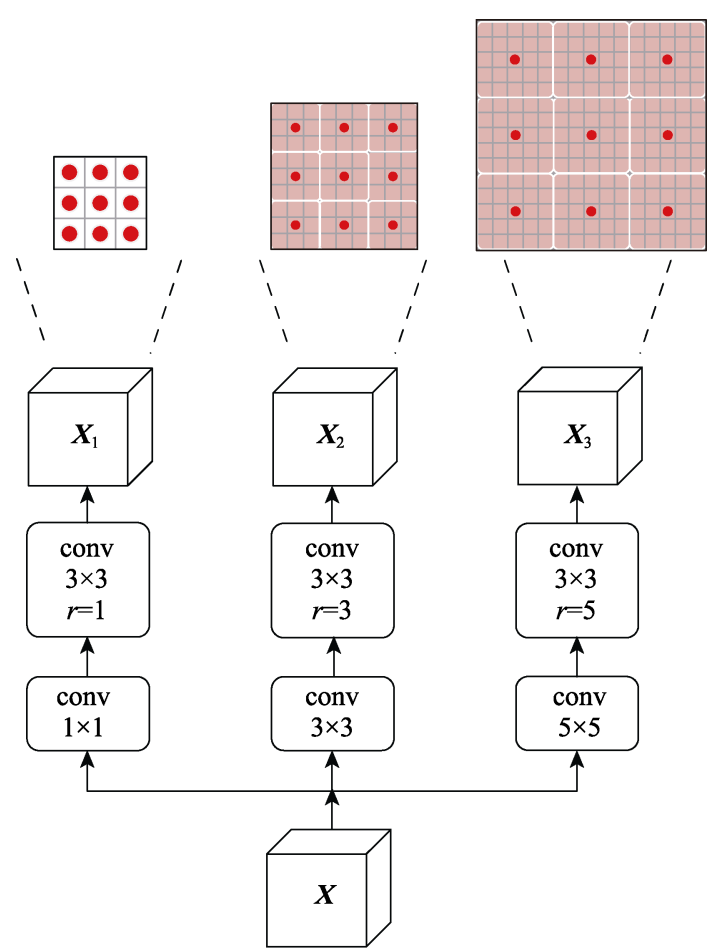

图 4 多分支并行空洞卷积结构

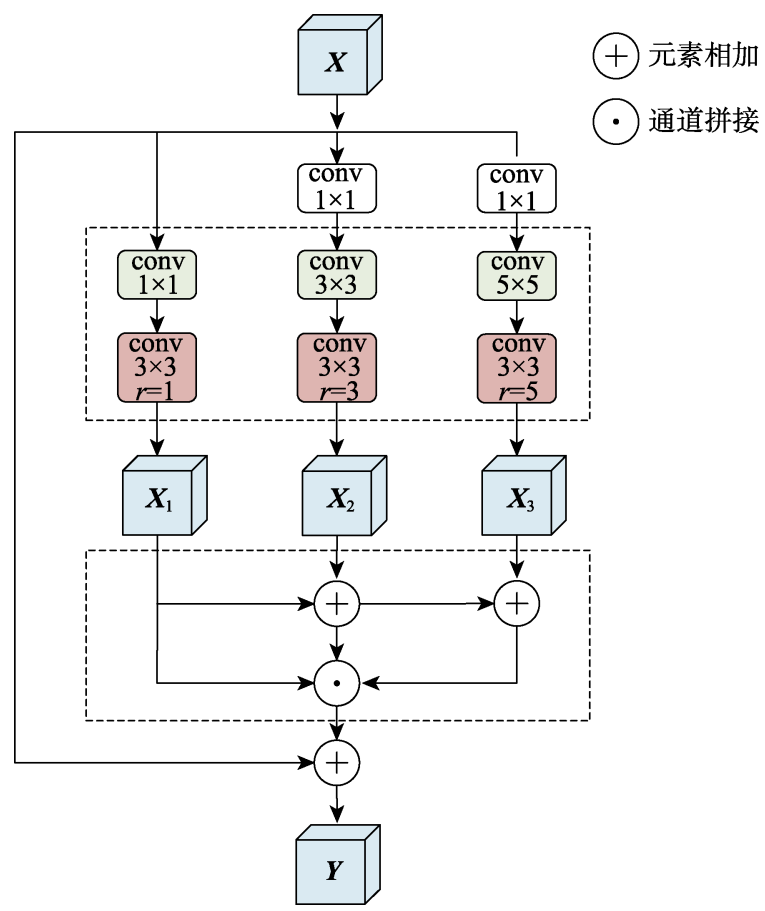

图 5 多分支并行空洞卷积总体结构

*为卷积操作; $C_{1 \times 1}, C_{3 \times 3}$ 和 $C_{5 \times 5}$ 分别为核大小为 $1 \times 1,3 \times 3$ 和 $5 \times 5$ 的普通卷积; $\boldsymbol{D}_{r=1}, \boldsymbol{D}_{r=3}$ 和 $\boldsymbol{D}_{r=5}$ 分别为核大小为 $3 \times 3$, 扩张率为 $r=1, r=3$ 和 $r=5$ 的空洞卷积

本文在通道拼接前添加了逐级叠加的融合方 式 $\boldsymbol{Y}=\left\{\boldsymbol{X}_{1}, \boldsymbol{X}_{1}+\boldsymbol{X}_{2}, \boldsymbol{X}_{1}+\boldsymbol{X}_{2}+\boldsymbol{X}_{3}\right\}$, 其中, $\{\cdot\}$ 为通 
道拼接操作, 即从扩张率 $r=1$ 的空洞卷积提取的 特征开始进行逐像素相加, 使 $r=3$ 和 $r=5$ 支路的 特征不仅包含扩张率较大的空洞卷积提取的特征, 还包含扩张率较小的空洞卷积提取的小目标特征, 从而改善网络对不同尺度特征的提取效果.

\section{2 非局部化结构}

非局部化的思想源于非局部网络，该网络主 要用于处理视频和音频中的长程依赖关系, 在视 频分类和机器翻译中取得了不错的效果. 虽然图 像检测并不具备时间上的依赖关系，但图像中的 像素点之间不是孤立存在的, 某像素点与其他像 素点之间不是孤立存在的, 某像素点与其他像素 点之间存在某种关联. 因此, 本文将上述思想引人 图像检测中，用于计算图像中像素点之间的空间 依赖关系. 该结构通过计算低层特征图中某像素 点与其他像素点的相似性, 并进行加权求和后表 示该像素点的特征信息, 从而捕获每个像素点特 征的全局信息，增强不同像素之间的联系，达到增 强低层特征上下文信息的目的.

非局部化结构如图 6 所示, 公式表示为

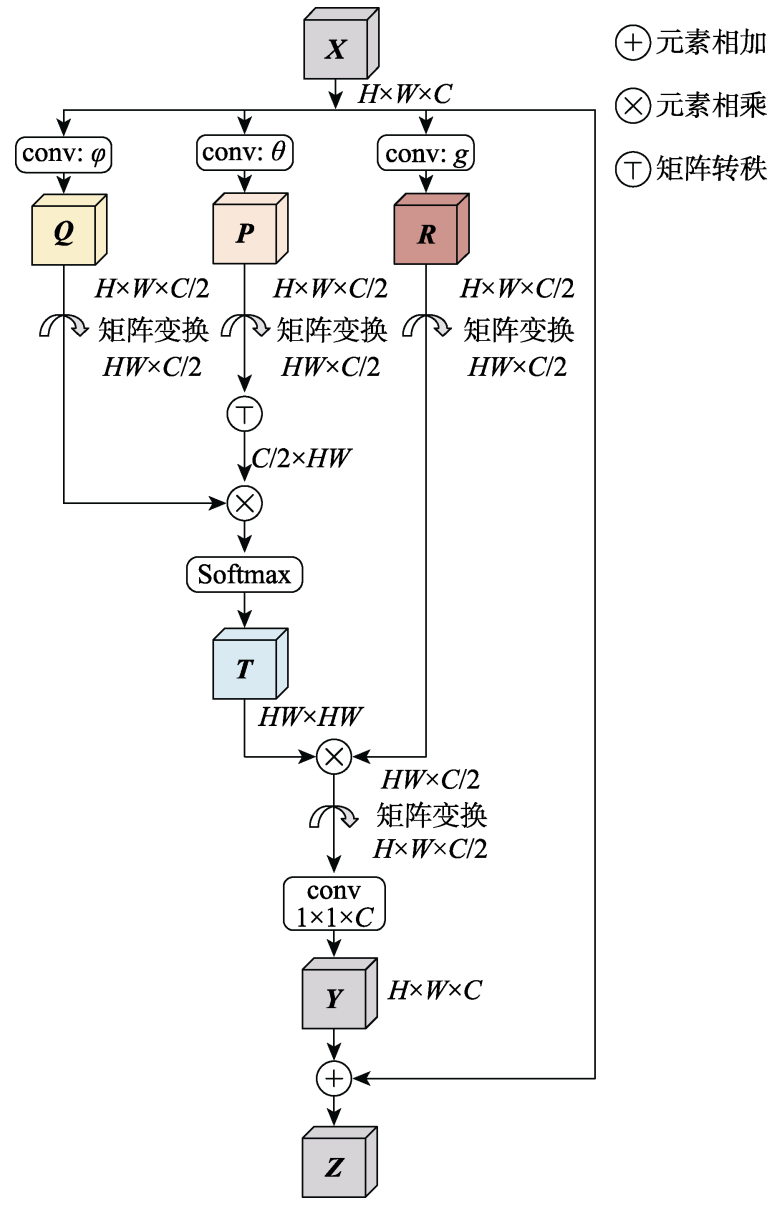

图 6 非局部化结构

$$
\boldsymbol{Y}_{j}=\operatorname{Softmax}\left(\theta\left(\boldsymbol{X}_{i}\right)^{\mathrm{T}} \varphi\left(\boldsymbol{X}_{j}\right)\right) g\left(\boldsymbol{X}_{j}\right)
$$

其中, $\boldsymbol{X}$ 和 $\boldsymbol{Y}$ 分别为输人和输出特征, 大小均为 $H \times W \times C ; i$ 为输人特征图上某个像素的索引; $j$ 为输人特征图上所有像素的索引. $\theta\left(\boldsymbol{X}_{i}\right)=\boldsymbol{W}_{\theta} \boldsymbol{X}_{\boldsymbol{i}}$, $\varphi\left(\boldsymbol{X}_{j}\right)=\boldsymbol{W}_{\varphi} \boldsymbol{X}_{j}, g\left(\boldsymbol{X}_{j}\right)=\boldsymbol{W}_{g} \boldsymbol{X}_{j}$ 为卷积操作, $\boldsymbol{W}_{\theta}$, $W_{\varphi}$ 和 $W_{g}$ 为学习得到的矩阵权重. 通过卷积 $\theta$ 和 $\varphi$, 可以获得矩阵 $\boldsymbol{P}$ 和 $\boldsymbol{Q}$, 大小均为 $H \times W \times C / 2$. 将矩阵 $\boldsymbol{P}$ 与 $\boldsymbol{Q}$ 进行相乘得到自相关矩阵, 即得到 特征图上某像素 $i$ 与所有像素 $j$ 的相关性. 由于相 乘后的矩阵与原矩阵的数值差异, 需要使用 Softmax 函数对其进行归一化处理, 得到 $H W \times$ $H W$ 大小的相关性矩阵 $\boldsymbol{T}$. 为了将相关性体现到 每个通道上, 本文引人卷积 $g$, 得到矩阵 $\boldsymbol{R}$. 将矩 阵 $\boldsymbol{R}$ 进行矩阵变换后与相关性矩阵 $\boldsymbol{T}$ 相乘, 得到 $H W \times C / 2$ 大小的矩阵, 即得到每条特征通道的相 关性. 最后, 通过矩阵变换以及卷积操作将其转化 为与输人特征 $\boldsymbol{X}$ 相同大小的特征矩阵 $\boldsymbol{Y}$. 结合残 差网络, 非局部化模块表示为 $\boldsymbol{Z}=\boldsymbol{W}_{\boldsymbol{Z}} \boldsymbol{Y}+\boldsymbol{X}$, 其中 $W_{Z}$ 为学习的权重矩阵.

\section{2 实验结果与分析}

\section{1 实验方法与设置}

为了更好地验证本文算法对不同类别物体以 及不同尺度大小物体检测的有效性, 分别在 PASCAL VOC 数据集 ${ }^{[12]}$ 和 MS COCO 数据集 ${ }^{[13]}$ 上 进行实验. 采用的评价指标与目前流行算法一致, 在 PASCAL VOC 数据集中, 采用平均精度均值 (mean average precision, mAP) 作为评价指标. 在 MS COCO 数据集中, 采用平均准确率 (average precision, $\mathrm{AP}$ )作为评价指标. 其中, $\mathrm{AP}_{\mathrm{IoU}}$ 为不同 交并比值(intersection over union, IoU)的平均准确 率, $\mathrm{AP}_{\text {area }}$ 为不同尺度目标的平均准确率.

在训练时, 为了增强模型的性能, 本文采用与 SSD 类似的数据增强方法, 包括随机裁剪、随机尺 度变换、随机水平和垂直翻转等. 由于本文在网络 的低层进行了多尺度特征提取, 因此在特征图中 的每个像素点上生成 6 个包围框, 包括 2 个不同大 小的正方形包围框、纵横比为 0.5 的不同大小的长 方形包围框，以及纵横比为 2 的 2 个不同大小的长 方形包围框, 以满足多尺度特征的要求. 每层的包 围框数量分别设置为 $6,6,6,6,4$ 和 4 . 其中, 前 4 层包围框设置相同, 后 2 层设置与 SSD 算法包围 
框设置相同.

为了得到更合适的空洞卷积扩张率，进行不 同大小空洞卷积扩张率的对比实验. 实验以 ASPP 结构的扩张率组合 $R=(6,12,18)$ 为基础，并将扩张 率分别降低为 $R_{1}=(3,5,10)$ 以及 $R_{2}=(1,3,5)$ 作为 对比. 实验的数据集为 PASCAL VOC 2007, 输人 图像大小为 $320 \times 320$, 实验结果如表 1 所示. 可以 看出, 随着扩张率的逐步降低, 图像中占比较大的 物体类别(如 bike, bus, cat 和 train)的检测精度逐渐 降低，小物体类别(如 aero, bird, bottle 和 person)的 检测精度逐渐增加. 而其他物体类别(如 boat, mike 和 sofa 等)由于在图像中占比居中, 在扩张率中等 的 $R_{1}$ 时精度较高. 可见，扩张率会影响不同尺度 大小物体的检测效果. 综上所述，本文在下面的实 验中采用平均检测精度较高的 $R_{2}$ 作为多分支并行 空洞卷积结构的扩张率.

表 1 PASCAL VOC 2007 数据集上 不同扩张率下的 $\mathbf{m A P}$

\begin{tabular}{|c|c|c|c|}
\hline \multirow{2}{*}{ 类别 } & \multicolumn{3}{|c|}{ 扩张率 } \\
\hline & $R$ & $R_{1}$ & $R_{2}$ \\
\hline aero & 84.9 & 85.2 & 86.0 \\
\hline bike & 86.3 & 84.3 & 85.1 \\
\hline bird & 74.3 & 80.6 & 81.9 \\
\hline boat & 71.1 & 74.6 & 73.2 \\
\hline bottle & 53.5 & 56.0 & 61.2 \\
\hline bus & 87.8 & 87.4 & 86.7 \\
\hline car & 86.5 & 86.6 & 86.3 \\
\hline cat & 89.9 & 88.1 & 86.1 \\
\hline chair & 63.6 & 62.3 & 63.9 \\
\hline cow & 86.1 & 84.8 & 84.7 \\
\hline table & 78.9 & 73.4 & 76.3 \\
\hline $\operatorname{dog}$ & 86.7 & 84.8 & 86.8 \\
\hline horse & 89.0 & 89.0 & 88.9 \\
\hline mbike & 87.9 & 88.1 & 82.4 \\
\hline person & 80.3 & 78.0 & 81.7 \\
\hline plant & 52.5 & 50.3 & 51.1 \\
\hline sheep & 81.7 & 81.4 & 82.3 \\
\hline sofa & 80.9 & 82.3 & 81.6 \\
\hline train & 89.2 & 88.6 & 87.4 \\
\hline tv & 79.7 & 78.6 & 81.5 \\
\hline $\mathrm{mAP}$ & 79.5 & 79.3 & 79.8 \\
\hline
\end{tabular}

注. 表中粗体表示最佳实验结果.

\subsection{PASCAL VOC 数据集实验结果}

本文在 PASCAL VOC 2007 和 PASCAL VOC 2012 数据集上进行网络模型的训练, 并在 PASCAL
VOC 2007 数据集上进行测试. 其中, 迭代次数为 $120 \mathrm{k}$ 次, 前 $80 \mathrm{k}$ 次学习率设置为 $10^{-3}, 80 \mathrm{k} \sim 100 \mathrm{k}$ 次学习率设置为 $10^{-4}, 100 \mathrm{k} 120 \mathrm{k}$ 次学习率设置为 $10^{-5}$, 训练的批次大小设置为 32 .

表 2 所示为本文算法与目前主流的目标检测 算法的对比结果, 可以看出, 在输人图片尺寸为 $320 \times 320$ 时，本文算法的检测精度为 $80.4 \%$, 比 SSD 算法和 DSSD 算法的检测精度分别提高了 $2.9 \%$ 和 $1.8 \%$, 并且有着几乎相同的检测速度. 另 外，和两阶段算法的 Faster R-CNN 相比, 本文算 法在输人图片像素尺寸为 $320 \times 320$ 的情况下，检 测精度超过了输入图片像素尺寸为 $1000 \times 600$ 的 Faster R-CNN算法. 与 YOLO 系列算法相比, 本文 算法在检测速度几乎相同的情况下，具有更高的 检测精度。

表 29 种算法在 PASCAL VOC 2007 数据集上 不同模型下的检测结果

\begin{tabular}{|c|c|c|c|c|}
\hline 算法 & 基础网络 & $\begin{array}{l}\text { 输人图片 } \\
\text { 像素尺寸 }\end{array}$ & $\mathrm{mAP} / \%$ & $\begin{array}{c}\text { 检测速度 } \\
\left(\text { 帧 } \cdot \mathrm{s}^{-1}\right)\end{array}$ \\
\hline \multirow{2}{*}{ Faster R-CNN ${ }^{[3]}$} & VGG & \multirow{2}{*}{$1000 \times 600$} & 73.2 & 7.0 \\
\hline & Residual-101 & & 76.4 & 2.4 \\
\hline $\operatorname{SSD}^{[5]}$ & VGG & $300 \times 300$ & 77.5 & 46.0 \\
\hline $\operatorname{DSSD}^{[14]}$ & Residual-101 & $321 \times 321$ & 78.6 & 9.5 \\
\hline $\operatorname{MDSSD}^{[10]}$ & VGG & $320 \times 320$ & 78.6 & 38.5 \\
\hline $\operatorname{STDN}^{[15]}$ & DenseNet-169 & $321 \times 321$ & 79.2 & 41.5 \\
\hline $\operatorname{Refinedet}^{[16]}$ & VGG & $320 \times 320$ & 80.0 & 40.3 \\
\hline YOLOv2 $2^{[17]}$ & Darknet-19 & $416 \times 416$ & 76.8 & 40.0 \\
\hline YOLOv3 $^{[18]}$ & Darknet-19 & $416 \times 416$ & 79.3 & 39.0 \\
\hline 本文 & VGG & $320 \times 320$ & 80.4 & 42.1 \\
\hline
\end{tabular}

注. 表中粗体表示最佳实验结果.

表 3 所示为当输人图片大小为 $320 \times 320$ 时, 本 文算法在 PASCAL VOC 2007 数据集上与其他单阶 段算法在不同类别上的实验结果对比. 本文算法 的 mAP 高于其他单阶段算法, 小目标类别(如 aero, car 和 plant 等)达到了其他算法的检测精度，bird， boat, sheep 和 person 等类别的检测精度均提高了 $1.0 \%$ 以上, 特别是 bottle 类的检测精度较其他算法 提高了 $10.6 \%$ 以上，这得益于本文算法对低层特征 的有效利用，弥补了单阶段算法对小目标检测效 果差的缺点.

图 7 所示为本文算法与 SSD 算法在 PASCAL $\mathrm{VOC}$ 数据集上的部分检测结果. 从前 2 列图片可 以看出，在同一图片中同时存在不同尺度大小的 物体时，本文算法要优于 SSD算法，能检测到 SSD 
表 36 种算法在 PASCAL VOC 2007 数据集上

\begin{tabular}{|c|c|c|c|c|c|c|}
\hline \multirow{3}{*}{ 类别 } & \multirow{2}{*}{\multicolumn{6}{|c|}{ 不同类别下的 mAP }} \\
\hline & & & & & & \\
\hline & $\mathrm{SSD}^{[5]}$ & $\operatorname{DSSD}^{[14]}$ & $\operatorname{MDSSD}^{[10]}$ & $\mathrm{STND}^{[15]}$ & YOLOv2 $2^{[17]}$ & 本文 \\
\hline aero & 79.5 & 81.9 & 86.5 & 81.2 & 86.3 & 86.5 \\
\hline bike & 83.9 & 84.9 & 87.6 & 88.3 & 82.0 & 85.4 \\
\hline bird & 76.0 & 80.5 & 78.9 & 78.1 & 74.8 & 82.4 \\
\hline boat & 69.6 & 68.4 & 70.6 & 72.2 & 59.2 & 73.9 \\
\hline bottle & 50.5 & 53.9 & 55.0 & 54.3 & 51.8 & 65.6 \\
\hline bus & 87.0 & 85.6 & 86.9 & 87.6 & 79.8 & 86.5 \\
\hline car & 85.7 & 86.2 & 87.0 & 86.7 & 76.5 & 87.5 \\
\hline cat & 88.1 & 88.9 & 88.1 & 88.7 & 90.6 & 87.8 \\
\hline chair & 60.3 & 61.1 & 58.8 & 63.5 & 52.1 & 66.1 \\
\hline cow & 81.5 & 83.5 & 84.8 & 83.2 & 78.2 & 84.5 \\
\hline table & 77.0 & 78.7 & 73.4 & 79.4 & 58.5 & 76.4 \\
\hline $\operatorname{dog}$ & 86.1 & 86.7 & 84.8 & 86.4 & 89.3 & 84.2 \\
\hline horse & 87.5 & 88.7 & 73.4 & 89.3 & 82.5 & 87.9 \\
\hline mbike & 83.9 & 86.7 & 88.1 & 88.0 & 83.4 & 86.2 \\
\hline person & 79.4 & 79.7 & 78.0 & 77.3 & 81.3 & 82.3 \\
\hline plant & 52.3 & 51.7 & 52.3 & 52.5 & 49.1 & 53.4 \\
\hline sheep & 77.9 & 78.0 & 78.6 & 80.3 & 77.2 & 81.7 \\
\hline sofa & 79.5 & 80.9 & 74.5 & 80.8 & 62.4 & 80.3 \\
\hline train & 87.6 & 87.2 & 86.8 & 86.3 & 83.4 & 88.7 \\
\hline tv & 76.8 & 79.4 & 80.7 & 82.1 & 68.7 & 80.8 \\
\hline $\mathrm{mAP}$ & 77.5 & 78.6 & 78.6 & 79.3 & 76.8 & 80.4 \\
\hline
\end{tabular}

注. 表中粗体表示最佳实验结果.

算法漏检的目标. 从最后一列图片可以看出, 在图 片中仅存在小目标物体时，本文算法能检测到更 多的目标. 由此可见, 本文算法能有效检测图像中 不同尺度大小的目标，且能有效提升小目标的检 测效果.

\subsection{MS COCO 数据集实验结果}

MS COCO 数据集包含 80 种不同类别的物体, 超过 80000 张训练图片以及 20000 张测试图片. 在 该数据集中, 规定目标面积小于 $32 \times 32$ 的物体为 小目标，目标面积大于 $32 \times 32$ 且小于 $96 \times 96$ 的物体 为中等目标, 大于 $96 \times 96$ 的物体为大目标.

表 4 所示为本文算法在 MS COCO 测试集中的
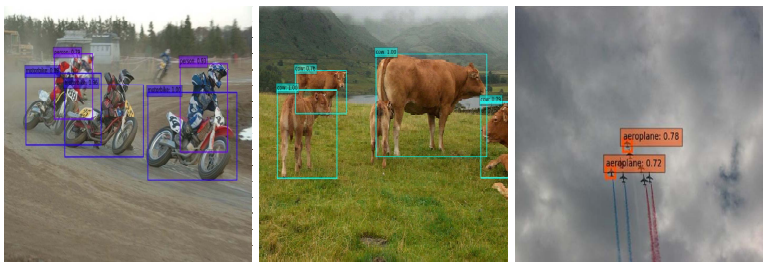

a. SSD 算法的检测结果

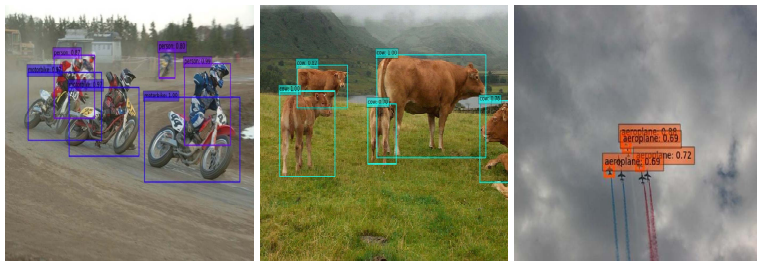

b. 本文算法的检测结果

图 7 PASCAL VOC 2007 数据集可视化结果对比

检测结果. 与两阶段算法 Faster R-CNN 相比, 本 文算法的检测效果更好. 与 R-FCN 算法相比, 本 文算法在输人图片尺寸更低的情况下，平均精度 高 $0.7 \%$ ，且对小目标的检测效果提升了 $1.6 \%$. 但 由于输人图片尺寸过小, 当 $\mathrm{IoU}$ 为 0.5 时, 检测精 度不如 R-FCN 算法. 与单阶段算法相比, 本文算 法比 DSSD 算法在大尺寸图像上的检测精度低 $0.6 \%$, 主要原因在于 DSSD 算法采用了比 VGG 基 础网络更深的 Resnet-101, 随着网络的加深, 高层 特征包含更多的语义信息，对于大尺寸物体的检 测效果更好，但与此同时增加了更多的计算量. 而 在小目标的检测精度上，本文算法检测精度比 DSSD 算法提高了 $5.0 \%$. 另外，与 SSD 算法相比, 平均检测精度提高了 $6.4 \%$, 不同尺度大小物体的 检测精度分别提高了 $5.8 \%, 7.1 \%$ 和 $5.6 \%$. 与 Refinedet ${ }^{[16]}$ 算法相比, 本文算法的平均精度和不 同尺度目标精度均高于 Refinedet 算法, 说明本文 算法可以有效地提取不同尺度物体的特征, 并增 强低层特征的上下文信息，验证了本文算法检测 多尺度目标的有效性.

表 48 种算法在 MS COCO 数据集上不同模型下的 AP

\begin{tabular}{|c|c|c|c|c|c|c|c|c|}
\hline \multirow{2}{*}{ 算法 } & \multirow{2}{*}{ 基础网络 } & \multirow{2}{*}{ 输人图片像素尺寸 } & \multicolumn{3}{|c|}{$\mathrm{AP}_{\mathrm{IoU}} / \%$} & \multicolumn{3}{|c|}{$\mathrm{AP}_{\text {area }} / \%$} \\
\hline & & & $0.5: 0.95$ & 0.5 & 0.75 & $\mathrm{~S}$ & $\mathrm{M}$ & $\mathrm{L}$ \\
\hline $\begin{array}{l}\text { Faster } \\
\text { R-CNN }^{[3]}\end{array}$ & VGG & $1000 \times 600$ & 24.2 & 45.3 & 23.5 & 7.7 & 26.4 & 37.1 \\
\hline $\mathrm{R}-\mathrm{FCN}^{[19]}$ & Residual-101 & $1000 \times 600$ & 29.9 & 51.9 & & 10.8 & 32.8 & 45.0 \\
\hline $\mathrm{SSD}^{[5]}$ & VGG & $300 \times 300$ & 25.1 & 43.1 & 25.8 & 6.6 & 25.9 & 41.4 \\
\hline $\operatorname{DSSD}^{[14]}$ & Residual-101 & $321 \times 321$ & 28.0 & 46.1 & 29.2 & 7.4 & 28.1 & 47.6 \\
\hline $\operatorname{STDN}^{[15]}$ & DenseNet-169 & $321 \times 321$ & 28.0 & 45.6 & 29.4 & 7.9 & 29.7 & 45.1 \\
\hline Refinedet $^{[16]}$ & VGG & $320 \times 320$ & 29.4 & 49.2 & 31.3 & 10.0 & 32.0 & 44.4 \\
\hline YOLOv2 $2^{[17]}$ & Darknet-19 & $416 \times 416$ & 21.6 & 44.0 & 19.2 & 5.0 & 22.4 & 35.5 \\
\hline 本文 ～～ & VGG & $320 \times 320$ & 30.6 & 49.9 & 31.6 & 12.4 & 33.0 & 47.0 \\
\hline
\end{tabular}

注. 表中粗体表示最佳实验结果; 0.5: 0.95 代表 10 个不同的 IoU 阈值( $0.5 / 0.05 / 0.95) ; 0.5$ 和 0.75 分别代表 IoU 取值为 0.5 和 0.75 ; S, M, L 分别代表小、中等和大目标. 


\section{4 消融实验}

为了进一步分析本文提出的结构对 SSD 算法 的提升效果，本文在低层特征上进行了不同的特 征图可视化结果对比实验. 图 8 所示为多分支并行 空洞卷积结构的可视化结果对比, 其中图 $8 \mathrm{a}$ 为原 图像, 图 $8 \mathrm{~b}$ 为 $\mathrm{SSD}$ 算法提取的特征图, 图 $8 \mathrm{c}$ 为本 文的多分支并行空洞卷积结构提取的特征图. 可 以看出，相比于 SSD 算法，本文的多分支并行空 洞卷积结构可以更好地提取不同尺度物体的特征.

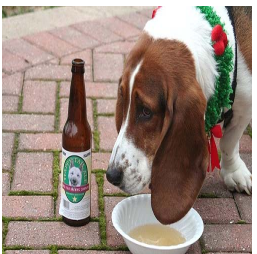

a. 原图像

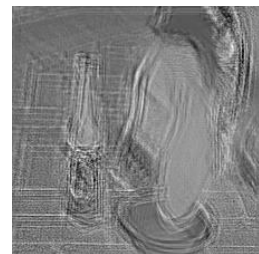

b. $\mathrm{SSD}^{[5]}$

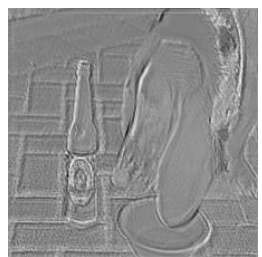

c. 本文
图 8 多分支并行空洞卷积结构可视化结果对比

为了说明非局部化结构的有效性，本文进行 了非局部化结构的可视化结果对比实验. 如图 9 所 示, 图 $9 a$ 为原图像, 图 $9 b$ 为 SSD算法提取的特征 图，图 9c 为本文的非局部化结构提取的特征图. 可以看出，在图像中同时存在不同尺度大小物体 时，采用非局部结构可以有效提取花盆的特征信 息，从而提高目标检测精度.

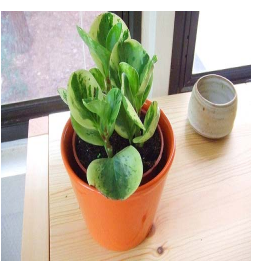

a. 原图像

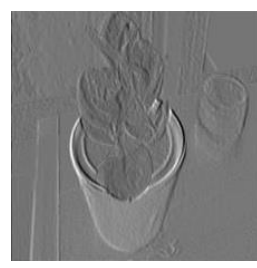

b. $\operatorname{SSD}^{[5]}$

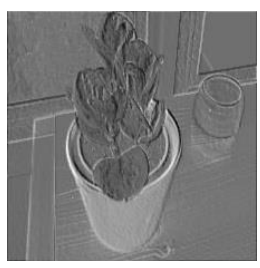

c. 本文
图 9 非局部化结构可视化结果对比

为了验证多分支并行空洞卷积结构和非局部 化结构对 SSD 算法的提升效果，本文在 PASCAL VOC 数据集中进行了不同模块的训练以及测试. 实验结果如表 5 所示, $\mathrm{SSD}$ 算法的 $\mathrm{mAP}=77.5 \%$, 将 非局部化结构单独嵌人 SSD 算法中, mAP 提升至

\section{表 5 PASCAL VOC 2007 数据集上不同结构下的 mAP}

\begin{tabular}{lc}
\hline \multicolumn{1}{c}{ 算法 } & $\mathrm{mAP} / \%$ \\
\hline $\mathrm{SSD}^{[5]}$ & 77.5 \\
$\mathrm{SSD}^{[5]}+$ 非局部化结构 & 78.7 \\
$\mathrm{SSD}^{[5]+}+$ 多分支并行空洞卷积结构 & 80.1 \\
本文 & $\mathbf{8 0 . 4}$
\end{tabular}

注. 表中粗体表示最佳实验结果.
$78.7 \%$ ，验证了非局部化结构的有效性. 将多分支 并行空洞卷积结构单独嵌人 SSD 算法中, mAP 提 升至 $80.1 \%$, 验证了多分支并行空洞卷积结构的有 效性. 将 2 个结构同时嵌人 SSD 算法中, mAP 提升 至 $80.4 \%$, 验证了本文算法的有效性.

\section{3 结 语}

本文在 SSD 算法的基础上，通过引人多分支 并行空洞卷积获取不同尺寸的感受野, 提高了网 络对不同尺度目标的特征提取能力. 同时, 采用非 局部化结构获取特征在空间域的全局信息，从而 增强特征的上下文信息. 在 PASCAL VOC 和 MS $\mathrm{COCO}$ 数据集上的实验结果表明, 本文算法有效 地提高了 SSD 算法对不同尺度物体的检测精度, 对小目标的检测效果明显超过其他流行算法, 并 且能够满足实时性的要求

\section{参考文献(References):}

[1] Girshick R, Donahue J, Darrell T, et al. Rich feature hierarchies for accurate object detection and semantic segmentation[C] //Proceedings of the IEEE Conference on Computer Vision and Pattern Recognition. Los Alamitos: IEEE Computer Society Press, 2014: 580-587

[2] Girshick R. Fast R-CNN[C] //Proceedings of the IEEE International Conference on Computer Vision. Los Alamitos: IEEE Computer Society Press, 2015: 1440-1448

[3] Ren S Q, He K M, Girshick R, et al. Faster R-CNN: towards real-time object detection with region proposal networks[J]. IEEE Transactions on Pattern Analysis and Machine Intelligence, 2017, 39(6): 1137-1149

[4] Redmon J, Divvala S, Girshick R, et al. You only look once: unified, real-time object detection[C]//Proceedings of the IEEE Conference on Computer Vision and Pattern Recognition. Los Alamitos: IEEE Computer Society Press, 2016: 779-788

[5] Liu W, Anguelov D, Erhan D, et al. SSD: Single shot multibox detector[C] //Proceedings of European Conference on Computer Vision. Heidelberg: Springer, 2016: 21-37

[6] Huang Jipeng, Shi Yinghuan, Gao Yang. Multi-scale Faster-RCNN algorithm for small object detection[J]. Journal of Computer Research and Development, 2019, 56(2): 319-327(in Chinese)

(黄继鹏，史颖欢，高阳. 面向小目标的多尺度 Faster-RCNN 检测算法 [J]. 计算机研究与发展, 2019, 56(2): 319-327)

[7] Li Xiaoguang, Fu Chenping, Li Xiaoli, et al. Improved faster R-CNN for multi-scale object detection[J]. Journal of Computer-Aided Design \& Computer Graphics, 2019, 31(7): 1095-1101(in Chinese)

(李晓光, 付陈平, 李晓莉, 等. 面向多尺度目标检测的改进 
Faster R-CNN 算法 [J]. 计算机辅助设计与图形学学报, 2019, 31(7): 1095-1101)

[8] Lin T Y, Dollár P, Girshick R, et al. Feature pyramid networks for object detection[C] //Proceedings of the IEEE Conference on Computer Vision and Pattern Recognition. Los Alamitos: IEEE Computer Society Press, 2017: 2117-2125

[9] Zhang Siyu, Zhang Yi. Small target pedestrian detection based on multi-scale feature fusion[J]. Computer Engineering \& Science, 2019, 41(9): 1627-1634(in Chinese) (张思宇，张轶. 基于多尺度特征融合的小目标行人检测 $[\mathrm{J}]$. 计算机工程与科学, 2019, 41(9): 1627-1634)

[10] Cui L S, Ma R, Lv P, et al. MDSSD: multi-scale deconvolutional single shot detector for small objects[J]. Science China (Information Sciences), 2020, 63(2): 98-100

[11] Chen L C, Papandreou G, Kokkinos I, et al. DeepLab: semantic image segmentation with deep convolutional nets, atrous convolution, and fully connected CRFs[J]. IEEE Transactions on Pattern Analysis and Machine Intelligence, 2018, 40(4): 834-848

[12] Everingham M, van Gool L, Williams C K I, et al. The PASCAL visual object classes (VOC) challenge[J]. International Journal of Computer Vision, 2010, 88(2): 303-338

[13] Lin T Y, Maire M, Belongie S, et al. Microsoft COCO: com- mon objects in context[C] //Proceedings of European Conference on Computer Vision. Heidelberg: Springer, 2014: 740-755

[14] Fu C Y, Liu W, Ranga A, et al. DSSD: deconvolutional single shot detector[OL]. [2020-08-01]. https://arxiv.org/pdf/1701. 06659.pdf

[15] Zhou P, Ni B B, Geng C, et al. Scale-transferrable object detection[C]//Proceedings of the IEEE Conference on Computer Vision and Pattern Recognition. Los Alamitos: IEEE Computer Society Press, 2018: 528-537

[16] Zhang S F, Wen L Y, Bian X, et al. Single-shot refinement neural network for object detection[C] //Proceedings of the IEEE Conference on Computer Vision and Pattern Recognition. Los Alamitos: IEEE Computer Society Press, 2018: 4203-4212

[17] Redmon J, Farhadi A. YOLO9000: better, faster, stronger[C] //Proceedings of the IEEE Conference on Computer Vision and Pattern Recognition. Los Alamitos: IEEE Computer Society Press, 2017: 6517-6525

[18] Redmon J, Farhadi A. YOLOv3: an incremental improvement [OL]. [2020-08-01]. https://arxiv.org/pdf/1804.02767.pdf

[19] Dai J F, Li Y, He K M, et al. R-FCN: object detection via region-based fully convolutional networks[C] //Proceedings of the 29th Annual Conference on Neural Information Processing Systems. New York: ACM Press, 2016: 379-387 